\title{
Burnout and Coping in the Perspective of Job Demands-Control-Support Model among Nurses
}

\author{
Abdelmajid Naceur*, Nawres Zriba \\ Laboratory EDIPS, University of Tunis, Tunis, Tunisia \\ Email: psynaceur@yahoo.fr
}

Received 4 June 2015; accepted 3 November 2015; published 6 November 2015

Copyright (c) 2015 by authors and Scientific Research Publishing Inc.

This work is licensed under the Creative Commons Attribution International License (CC BY). http://creativecommons.org/licenses/by/4.0/

(c) (i) Open Access

\begin{abstract}
The aims of the paper are to examine the effects of coping on burnout in the context of JD-C-S model. Background A postulate of the job demands-resources model is that two distinct yet related processes contribute to the development of burnout. The energetic process originates from demands and is mainly centered on emotional exhaustion; the motivational process originates from resources and is mainly centered on depersonalization. We postulated that coping and the job type have interaction effects on burnout.
\end{abstract}

\section{Keywords}

Burnout, Coping, Work Behaviors, Emotion, Motivation, Nursing

\section{Introduction}

During our observation of the workplace at the hospital; we looked closely at the attitude of nurses towards their patients; we identified a very interesting nurse's burnout phenomenon, which has led us, thereafter, to the present study.

Due to their occupation requirements and challenges, nurses come across many stressful situations such as exposure to death, risk of infections and conflicts with fellow health care workers due to inadequate staffing, work overload and irregular and extending working schedules. These distressing situations may also include dealing with the patients demands, answering their questions and easing the concerns of their families and, last but not least, dealing with violent patients; there have been indications that rates of severe injury and physical assault are high among healthcare workers (Bureau of Labor Statistics, 2002).

"Corresponding author. 
These stressors can be viewed as inherent to the profession of nursing all over the world, but what makes the Tunisian nurse's situation even worse is that their legal status is not settled, yet. Actually, they have been struggling for a long period to get their rights, and the Union Movement has been fighting in order to establish nursing specialties (Cardiac nursing, pediatric nursing...) to offer legal liability protection related to nursing practices and to increase the low pay.

At the end, it should be noted that repeated exposure to these chronic stressors may have a negative impact on one's emotional and mental health. The impact includes both personal and professional distress, loss of empathy, and poor health. Hence, we chose to study burnout among this particular population.

As for Maslach, burnout was conceptualized as a psychological syndrome in response to chronic emotional and interpersonal stress on the job and is most widely defined by the dimensions of exhaustion, depersonalization, and inefficacy (Maslach, Schaufeli, \& Leiter, 2001). She interviewed a wide range of human services workers about the emotional stress of their jobs. Hence, burnout research had its roots in care-giving and service occupations, in which the core of the job was the relationship between provider and recipient. So from the very beginning, burnout was studied not so much as an individual stress response but as an individual's response in an interpersonal context (Maslach et al., 2001).

We have decided to examine the effect of two critical factors considered to be determinants of the level of burnout. On the one hand and inspired by Karsek's job demand-control-support, studying the psychosocial job characteristics is warranted. On the other hand and because when confronted with any stressful life events, individuals normally have recourse to a wide range of coping strategies to modify the negative impact of distress and to enhance well being. Therefore we believed that it is relevant to emphasize the coping process. Research on coping over the past 30 years has been dominated by the work of Folkman and Lazarus (1988). The transacttional theory of Folkman and Lazarus identifies the process of coping, as critical mediators of stressful personenvironment relationships (Folkman, Lazarus, Gruen, \& DeLongis, 1986).

\section{Conceptual Framework}

Current theory and research on the relation between and indicators of adaptational status such as somatic health and psychological symptoms reflect the belief that this relation is mediated by coping processes.

\subsection{The Physiological Effect}

The earlier studies concerning this topic were heavily influenced by the physiological approach mostly envisaged by Selye the” founding father” of stress. For this author (Selye, 1977) stress is not something that we can avoid; it's a vital physiological nonspecific response of the body to any demand that increases the need for readjustment "Complete freedom from stress is death". Nonspecific means that different type of tasks, in fact almost any task could produce stress. Thus, when the body is exposed to continuous stress for long periods, it necessarily goes through the three phases of what Selye have called the General Adaptation Syndrome or GAS. The first stage, the alarm reaction, the second a stage of resistance and finally the stage of (Selye, 1977).

\subsection{The Cognitive Effect}

Later on the two concepts of stress and adjustment strategies was revised by the cognitive psychology. Through a transactional perspective (transactional model of stress and coping). Folkman and Lazarus (1988) at first, they were interested in the way emotion can impair with cognitive functioning as cognitive activity influences deployment of attention alter the subjective meaning of the encounter and enhance well being. For Folkman and Lazarus Stress is conceptualized as an interactional process, as we can’t limit it causes neither to an environmental nor to strictly individual sources. Therefore it is considered as the result of a dynamic relationship between the individual resources and the environmental requirements the stressful situation is than evaluated by a person as a threat to his well being exceeding his resources.

By this we mean that a perso-environment relationship is influenced by the person's characteristics (patterns of motivation, commitment, beliefs...), interpersonal relationships and the environmental requirement of the work place (Naceur \& Masmoudi, 2008). Later they made the assumption that the coping process may work in the following way: Once an event is appraised as stressful, that is, taxing a person's resources, it is assessed in terms of potential harm, loss, or challenge. Resources, whether psychological or material, derived from the self or others, are gathered and directed towards altering an event, transforming its implications (problem-focused 
strategies), or accepting the event where it is perceived to be out of control but trying to reduce the negative emotional affect caused by a stressor (emotion-focused strategies).

\section{Empirical Evidence}

Folkman and Lazarus (1986) conducted a study in which they have examined the relation between personality factors, primary appraisal (the stakes a person has in a stressful encounter), secondary appraisal (options for coping), somatic health status and psychological symptoms in a sample of 150 community-residing adults. The significant relations between appraisal, coping, and somatic health status were all negative, which indicated that the more subjects had at stake and the more they coped, the poorer their health was. In contrast, the more mastery they felt, the better their health was. They suggested three pathways through which coping might adversely affect somatic health status. First, coping can influence the frequency, intensity, duration, and pattern of neurochemical responses; second, coping can affect health negatively when it involves excessive use of damaging sub stances such as alcohol, drugs, and tobacco, or when it involves the person in activities of high risk to life and limb; and third, certain forms of coping (e.g., particularly denial-like processes) can impair health by alter adaptive health/illness-related behavior.

\subsection{The Social Psychological Effect}

Nurses used problem-focused coping in an attempt to change the stressful situation if this strategy was an option. For example, while nurses want to give excellent care, the resources are not always available. Emotion-focused coping, used to manage nurses' emotional response to the stressor, was used most often. This finding matches the research focusing on coping in health-related stressful situations. Social support coping was highly regarded and often used. Nurses expressed their feelings with each other and received validation from each other. Family and friends provided different forms of emotional and physical support. Several nurses also commented that "God" and "their" spiritual beliefs were sources of social support. A few indicated that their pets were a calming influence (Moszczynski \& Haney, 2002). Burnout occurs when professionals use ineffective coping strategies to try to protect themselves from work-related stress.

\subsection{Problem Solving Coping vs Emotion Focused Coping vs Social Support Coping}

It has been suggested that emotion focused coping was associated with a negative outcome and problem-solving strategy was associated with a positive outcome for hospital nurses or student nurses. The results indicated that problem focused coping is related to low exhaustion and cynicism, and high professional efficacy. This suggested that problem solving focused coping is associated to a positive outcome for men. On the other hand, problem solving was associated with both positive and negative outcomes, such as professional efficacy and exhaustion, in women. Although the methods of problem-solving training are often included in a cognitive-be- havioral approach (Naceur, 2010), these findings suggest that the use of problem solving as a coping strategy may be a double-edged sword, especially for women. On the other hand, problem solving coping was not asso- ciated with burnout in men. When conducting interventions with nurses, we should take these gender differences into account (Sasaki, Kitaoka-Higashiguchi, Morikawa, \& Nakagawa, 2009).

Initiatives to promote social support and to find solutions for a problem are likely to have the more immediate benefits in enhancing a person's well-being and avoiding burnout (Gibbons, 2010). Perceived social support has been related to the use of adaptive coping strategies and to lower levels of burnout (Boscarino et al., 2004; Brown \& O’Brien, 1998; McRaith \& Brown, 1991). Cited by (Baker, O’Brien, \& Salahuddin, 2007).

\subsection{Coping, Job Stress and Psychosocial Demand-Control Model}

Studies were promising, finding that low decision latitude, whether through self report or independent assessment, predicted a high level of stress and higher rates of short term and long term sickness absence. And in women high demands predicted poor health functioning. People with high demands, and to a low control, are at increased risk for heart disease (Kuper \& Marmot, 2003). Furthermore it has been proven that perceived workload and time pressures are strongly and consistently related to burnout (Abel \& Sewell, 1999; Houkes, Janssen, de Jonge, \& Nijhuis, 2001; Lee \& Ashforth, 1996). In addition, lack of social support at work has been found to increase levels of burnout (Houkes et al., 2001; Peeters \& Le Blanc, 2001) Cited by M’kikangas et al. 
The Karasek's theory affirmed that workers who have simultaneous low decision latitude and high demands cannot restrain the stress caused by the high demands through time management or learning new skills, and so become subject to high stress at work and are at increased risk of disease. It is therefore the constraints on decision making, together with high demands, which produce the harmful condition of stress at work, or "job strain". (Kuper \& Marmot, 2003). He hypothesized that job demands (e.g. high workload) were not in themselves harmful but, when combined with low employee control, these demands could lead to the development of cardiovascular disease. When job demands and control are both high Karasek describes the job as "active", that is, one in which the demands act as sources of challenge rather than as sources of mental and physical stress (Dwyer \& Ganster, 1991).

\section{The Present Study}

Some of the previous studies examined the relationship between either burnout and coping strategies or burnout and the work's psychological demand, others more recent studies have argued the value of active coping or problem focused coping in a job strain context. However no comprehensive review has proposed a potential influence of both coping strategies and psychosocial workload on the level of burnout.

At the beginning we were interested in witch way coping can affect the level of burnout but giving the emotional and mental workload of nurses we have decided to study this relation in the both cognitive and psychosocial perspective to try to understand the subject's cognitive and behavioral efforts to handle a psychologically demanding job.

It goes without saying that in the case of a non demanding job (passive job) there will be no evidence of a burnout therefore we will be only interested in 3 types of jobs: the job causing strain, the active kind of job and the low strain job.

Hypothesis 1: A high level of burnout will be associated with emotion focused coping in a context of high extent job demand and low decision latitude. (High job strain)

Hypothesis 2: A low level of burnout will be associated with problem focused coping in a context of high extent job demand and high sense of control. (Active job situation)

Hypothesis 3: A low level of burnout links to social support coping in a context of low demand and high control. (Low job strain)

\subsection{Method}

\subsubsection{Participants}

Participants were 72 nurses. The majority of them were female nurses (81.94\%), their mean age were 39.88 (SD =11.46).

The participants were well educated; the majority of them hold a college degree and they were also separated to 3 groups according to their seniority, divided as follows (Figure 1):

- Group 1: 0 to 5 years seniority , composed of 22 nurses

- Group 2: 6 to 10 years seniority, composed of 7 nurses

- Group 3: over 10 years seniority, composed of 43 nurses

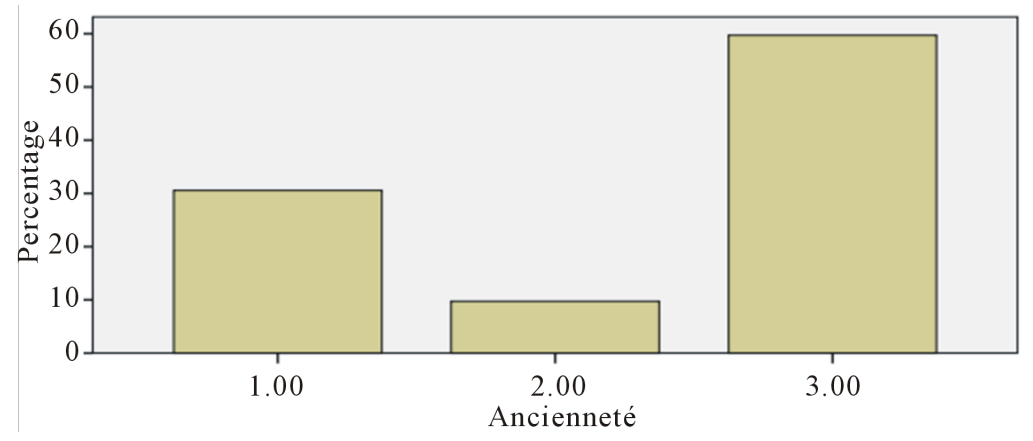

Figure 1 . The percentage of the different seniority groups among nurses. 


\subsubsection{Procedure}

Three Arabic translated questionnaires measuring the level of burnout the coping strategy and the job content, were distributed to over 80 nurses working in the "Habib Thameur Hospital" from different wards. I have explained to them that the study was confidential and anonymous. Also the purpose of the study was briefly described.

\subsubsection{Measures}

1) Masalch Burnout Inventory

In the present study, the MBI is used to measure burnout; the Maslach Burnout Inventory (MBI) became the almost universally accepted 'gold standard' to assess burnout, high scores on exhaustion and cynicism, and low scores on professional efficacy, are indicative of burnout.

The instrument is made up of 22 items utilizing a 7 point Likert scale going from 0: never to 6: daily.

The MBI includes three subscales:

EE: includes 9 items $(1,2,3,6,8,13,14,16,18)$

DP: contain 5 items $(5,10,11,15,22)$

PA: consisted of 8 items: $(4,7,9,12,17,18,19,21)$

The measure has been demonstrated to be a valid and reliable instrument, with good internal consistency coefficients (Cronbach's alpha $\geq 0.90$ ) and 1 - 4 month test-retest coefficients ranging from 0.66 to 0.89 .

The range of burnout experienced is broken down as follows: EE (low, $\leq 16$; average, 17 - 26; high $\geq 27$ ); DP (low, $\leq 6$; average, 7 - 12; high, $\geq 13$ ); and PA (low, $\geq 39$; average, 38 - 32; high, $\leq 31$ ). Burnout is associated with higher scores on the EE and DP subscales and a lower score on the PA subscale. (Hoge, 2009).

2) Karsek's job content questionnaire

For the 29 item version of the JCQ (1997) used in this study 3 dimensions were measured

- Decision latitude (9 items): divided to two subscales

* Decision autonomy (Q1, Q2, Q3, Q4, Q5, Q6)

* Use of skills and qualifications (Q7 Q8 Q9)

- Psychological demand contain 9 items: (Q10, Q11, Q12, Q13, Q14, Q15, Q16, Q17, Q18)

- Social support (11 items) composed of

* Co-worker social support (Q19, Q20,Q21, Q22, Q23, Q24)

* Supervisor support (Q25, Q26, Q27, Q28, Q29)

Subject's answers are rated on a 4 point likert scale "strongly disagree”, "disagree”, "agree”, and "strongly agree" coded simultaneously from 1 to 4 .

The JCQ is a valid and a reliable instrument due to its international calibration it was also translated to many languages such as English, French, Japanese, Italian Swedish German, etc.

The questionnaire was translated in Arabic. The internal consistency coefficients of the Arabic version of the JCQ measured by the Cronbach's alpha (0.90) (Hafsi, 2013).

A low score on the job demand $<21$ is an evidence of a high job demand

A score on the decision latitude $<70$ indicate a high level of control

3) Ways of Coping Check List

The French Ways of Coping Checklist WCC-R (Cousson et al., 1996) is a 27-item coping scale assessing three coping strategies (problem-focused coping; emotion-focused coping; and seeking social support) and derived from Lazarus and Folkman's WCC, Bruchon-Schweitzer, Cousson, Quintard, \& Nuissier in 1996 administered the French version of the WCC validated by Vitaliano Russo, Carr, Maiuro, \& Becker, (1985) to 468 adult subjects and found a three factor solution: problem-focused; emotion-focused; and social-support seeking. The revised instrument contained a 27-item French version of the WCC with good internal consistency, test-retest reliability, and construct and criterion validities. Respondents use a 4-point Likert-type scale ranging from No to Yes.

\section{Results}

\subsection{Preliminary Analyses}

\subsubsection{Coping}

Table 1 presents the means and standard deviations. 


\subsubsection{Burnout}

Using the MBI we found high level of Emotional exhaustion (EE mean score of 28.2; SD = 11.58) and depersonalization (DP mean score of 7.3; $\mathrm{SD}=6.76$ ), with a low range on personal accomplishment (PA mean score of 32.8; SD = 7.2). These findings were consistent with previous studies. Utilizing the MBI categorization data for mental health workers Paris Jr. and Hoge found these following outcomes, emotional exhaustion (mean score of 19.99; $\mathrm{SD}=9.83$ ) and depersonalization (mean score of 5.21 ; $\mathrm{SD}=4.26$ ) were in the average range, while PA was in the low (mean score of 41.64 ; $\mathrm{SD}=4.78$ ).

Participants were experiencing work-related distress. 6.9\% of the participants suffered from high levels of burnout, $13 \%$ experienced moderate levels of burnout, while the majority of them (48.6) tolerated low levels of burnout. Although 26, 3\% of our subjects showed no sign for a work related distress as defined by Maslach et al. (2001).

\subsubsection{Job Content}

The frequency and the percentage of the 4 types of job presented by Karasek are reported in Table 2.

As for the descriptive statistics it is reported in Table 3.

\subsection{Test of Hypotheses}

\subsubsection{Multiple Analyses Regression}

Using the SPSS we try to analyze the prediction value of the interaction between coping strategies and job types. $R 2=0.982, R=0.991$. A value of 0.991 , in this example, indicates a good level of prediction. The ANOVA Table 4 indicates $\mathrm{F}(3,68)=16.94, p<0.05$.

Table 1. Means and standard deviations of Coping $(N=72)$.

\begin{tabular}{ccc}
\hline Coping & $\boldsymbol{M}$ & SD \\
\hline Problem focused coping & 31.68 & 5.44 \\
Emotion focused coping & 23.48 & 5.6 \\
Social support focused coping & 21.93 & 5.28 \\
\hline
\end{tabular}

Table 2. Frequency and percentage of Kazrasek’s Job types $(N=72)$.

\begin{tabular}{ccc}
\hline Type of Job & Frequency & $\%$ \\
\hline Active & 17 & 23.6 \\
Low strain & 19 & 26.3 \\
Passive & 17 & 23.6 \\
Strain & 19 & 26.3 \\
\hline
\end{tabular}

Table 3. Descriptive statistic for the job C-D-S model $(N=72)$.

\begin{tabular}{cccc}
\hline Job & $\boldsymbol{M}$ & Median & SD \\
\hline DL & 48.92 & 49.00 & 10.22 \\
DE & 19.81 & 19.50 & 4.13 \\
SS & 25.67 & 25.00 & 5.31 \\
\hline
\end{tabular}

Table 4. Multiple analyses regression $(N=72)$.

\begin{tabular}{cccccc}
\hline & B & E S & B & T & sig \\
\hline (Constante) & 1.05 & 0.014 & & 75.24 & 0.00 \\
coping_centred & -0.00 & 0.01 & -0.00 & -0.41 & 0.68 \\
work_centred & 0.97 & 0.01 & 0.98 & 57.76 & 0.00 \\
work_coping_centred & 0.01 & 0.02 & 0.01 & 0.87 & 0.38 \\
\hline
\end{tabular}

a. Variable dependant: burnout. 
Job type (work) and coping strategy interaction (work_coping_centred) was not significant $(\beta=0.015, p>$ 0.05 ). However, the other variable, type of job (work_centred) was a good predictor for the level of burnout. ( $\beta$ $=0.988, p<0.05)$. Unlike the coping strategy variable $(\beta=-0.413, p>0.05)$. Our null hypotheses is accepted.

\subsubsection{Correlation Analyses}

Table 5 presents correlations between the scales.

Table 5 demonstrate that there is a strong relation between type of job and the level of burnout, while there is no relationship between a certain coping strategy and the level of burnout but correlation value of the interaction between both of the variables with the burnout phenomenon is $r=0.265$.

1) Coping and burnout

There was a weak negative relation between emotional exhaustion and problem focused coping $r=-0.059$, a weak positive relation between emotional exhaustion and emotional focused coping $r=0.154$ and a weak positive relation between emotional exhaustion $r=-0.061$.

We found a weak negative relation between depersonalization and problem focused coping $r=-0.032$, a strong positive relation between depersonalization and emotion focused coping $r=0.215$ and a negligible relationship between depersonalization and social support seeking coping $r=0.132$. Strong relation between personal accomplishment and problem focused coping $r=0.303$, no relation between personal accomplishment and emotion focused coping $r=0.090$ and a strong relation between personal accomplishment and a strong relation between personal accomplishment and social support focused coping.

2) Burnout and control-demand-support model

We found a strong positive relationship between emotional exhaustion and psychological demand $\mathrm{r}=-0.24$. There is no relation between emotional exhaustion and decision latitude $r=0.083$ and a weak negative relationship between emotional exhaustion and social support $r=0.146$.

- A strong negative relation between depersonalization and job control $r=0.215$

- A strong positive relation between depersonalization and social support $r=-0.368$

3) Coping and control-demand-support model

We found:

- No relationship between problem focused coping and job demand $r=0.09$

- No relationship between problem focused coping and decision latitude $r=-0.028$

- No relationship between problem focused coping and social support $r=-0.096$

About Emotion focused coping and control-demand-support model, we identified:

- A weak negative relation between emotion focused coping and decision latitude $r=0.144$

- A negligible relation between emotion focused coping and work psychological demand $r=0.072$

- A negligible relation between emotion focused coping and social support $r=0.05$

From the other side, the results shows no relationship between social support coping and decision latitude $r=$ -0.059 and a weak negative relation between social support and decision latitude $r=0.148$. We found also a weak positive relation between social support coping and social support from the work job.

Table 5. Correlations between the scales $(N=72)$.

\begin{tabular}{|c|c|c|c|c|c|c|c|}
\hline & 1 & 2 & 3 & 4 & 5 & 6 & 7 \\
\hline \multicolumn{8}{|l|}{ 1) Coping } \\
\hline 2) Work & 0.10 & & & & & & \\
\hline 3) Burn & 0.09 & $0.99^{* *}$ & & & & & \\
\hline 4) Coping-Work & $0.50^{* *}$ & $0.82^{* *}$ & $0.82^{* *}$ & & & & \\
\hline 5) Work-centred & 0.10 & 0.01 & $0.99^{* *}$ & $0.82^{* *}$ & & & \\
\hline 6) Work-coping-centred & -0.00 & $0.25^{*}$ & $0.26^{*}$ & $0.55^{* *}$ & $0.25^{*}$ & & \\
\hline 7) Coping-centred & 0.01 & 0.10 & 0.09 & $0.50^{* *}$ & 0.10 & -0.00 & \\
\hline
\end{tabular}

${ }^{*} p<0.05 ;{ }^{* *} p<0.01$. 


\section{Discussion}

The aim of the current study was to examine the effects of coping on burnout in the context of the JD-C-S model. We expected that coping and the job type have interaction effects on burnout.

Contrary to our expectations, coping and the job type had independent and direct effects on burnout and no effects of any interaction between them were found. That is, the relationship relation between coping and burnout did not change regardless of how much a job can be psychologically demanding. Therefore, it is reasonable to reject our hypotheses.

The type of job is more likely associated with the level of burnout.

According to Maslach, workload is most directly related to the exhaustion aspect of burnout. In fact our results confirmed the strong positive relationship between the job psychological demand and the emotion exhaustion from one hand and with depersonalization from the other plus a positive relationship between personal accomplishment and decision latitude also there were a negative relationship between emotional exhaustion and social support.

Emotion focused coping was significantly and positively correlated with emotional exhaustion.

Whereas there have been no significant relation between problem solving coping and social support seeking strategies with the level of burnout this result is in contradiction to the earlier mentioned studies. From these findings, both these strategies seem to have no adaptive function.

As Shimazu announced that job demands were positively correlated with age, supervisor support, active coping, and psychological distress. Each job resource variable (job control, supervisor support, and coworker support) was positively correlated with active coping and negatively correlated with psychological distress. However in the present study, some inconsistent findings were indicated as we found no relationship between the different coping strategies and the psychological demand of a work place, neither with the level of burnout.

\section{Conclusion}

Many studies have addressed the manner of burnout among health care workers. Results were fascinating, according to some authors, some $25 \%$ of all nurses suffer from burnout (Landau, 1992). 5\% of our population presented a high level of burnout. Studies have also shown the importance of psychosocial risks for physical and mental health (Roland-Lévya, Lemoinea, \& Jeoffrionb, 2014) particularly in the medical sector and among hospital workers. Therefore we conducted an investigation of over 80 nurses and we tried to evaluate the role of coping, moderated by the perspective of job satisfaction in affecting the level of burnout.

We stumbled upon a rather intriguing result. It seems that it doesn't matter how a person may cope with the stressful situation, using a certain coping strategy does not lead to a positive nor to a negative outcome on one's mental health. So the level of burnout doesn't depend on a certain coping strategy. Whereas, the type of job has a direct effect and it is strongly associated to burnout.

No interaction between coping and type of job has been found.

There were some limitations to the present study. First, we used 2 non standardized questionnaires, the WCC and the MBI, so cultural factors may affect the results besides a self reported questionnaire may not be the best way to assess psychological distress; future researches can use the CISS to assess coping strategies questionnaire or the GCQ to asses job stress. We also wanted to conduct a multiple regression test between all the dimensions of all our variables, we could not achieve this goal with the little knowledge we have on SPSS.

\section{References}

Baker, M., O’Brien, M., \& Salahuddin, M. (2007). Are Shelter Workers Burned Out?: An Examination of Stress, Social Support, and Coping. Journal of Family Violence, 22, 465-474. http://dx.doi.org/10.1007/s10896-007-9103-1

Dwyer, D. J., \& Ganster, D. C. (1991). The Effects of Job Demands and Control on Employee Attendance and Satisfaction. Journal of Organizational Behavior, 12, 595-608. http://dx.doi.org/10.1002/job.4030120704

Folkman, S., \& Lazarus, R. (1987). Transactional Theory and Research on Emotions and Coping. European Journal of Personality, 1, 141-169. http://dx.doi.org/10.1002/per.2410010304

Folkman, S., \& Lazarus, R. (1988). The Relationship between Coping and Emotion Implication for Theory and Research. Social Science and Medicine, 26, 309-317. http://dx.doi.org/10.1016/0277-9536(88)90395-4

Folkman, S., Lazarus, R., Gruen, R., \& DeLongis, A. (1986). Appraisal, Coping, Health Status, and Psychological Symptoms. Journal of Personality and Social Psychology, 50, 571-579. 
Hafsi, A. (2013). Condition de travail,qualité de vie et santé psychologique chez les enseignants des collèges du grand Tunis. Thèse de doctorat soutenue le 17 décembre 2013 à FSHST.

Houkes, I., Janssen, P. P. M., de Jonge, J., \& Nijhuis, F. J. N. (2001). Work and Individual Determinants of Intrinsic Work Motivation, Emotional Exhaustion, and Turnover Intention: A Multi-Sample Analysis. International Stress Management, 8, 257-283. http://dx.doi.org/10.1023/A:1017561531748

Kuper, H., \& Marmot, M. (2003). Job Strain, Job Demands, Decision Latitude, and Risk of Coronary Heart Disease within the Whitehall II Study. J Epidemiol Community Health, 57, 147-153. http://dx.doi.org/10.1136/jech.57.2.147

Maslach, C., Schaufeli, W., \& Leiter, P. (2001). Job Burnout. Annual Review of Psychology, 52, 397-422. http://dx.doi.org/10.1146/annurev.psych.52.1.397

Moszczynski, B., \& Haney, J. (2002). Stress and Coping of Canadian Rural Nurses Caring for Trauma Patients Who Are Transferred Out. Journal of Emergency Nursing, 28, 496-504.

Naceur, A. (2010). Les traces émotionnelles dans la prise de décision. In S. Masmoudi, \& A. Naceur (Eds.), Du percept à la décision. Intégration de la cognition, l'émotion et la motivation. Bruxelles: De Boeck.

Naceur, A., \& Masmoudi, S. (2008). Peut on comprendre le fonctionnement cognitif humain sans comprendre ce qui l'oriente? In A. Naceur, \& S. Masmoudi (Eds.), Cognition-Emotion-Motivation. Intégrer... Mieux expliquer la performance. Tunis: CNIPRE.

Paris Jr., M., \& Hoge, A. (2009). Burnout in the Mental Health Workforce: A Review. Journal of Behavioral Health Services \& Research, National Council for Community Behavioral Healthcare.

Peeters, M. C. V., \& Le Blanc, P. M. (2001). Towards a Match between Job Demands and Sources of Social Support: A Study among Oncology Care Providers. European Journal of Work and Organizational Psychology, 10, 53-72. http://dx.doi.org/10.1080/13594320042000034

Roland-Lévya, C., Lemoinea, J., \& Jeoffrion, C. (2014). Health and Well-Being at Work: The Hospital Context. Revue européenne de psychologie appliquée, 64, 53-62.

Sasaki, M., Kitaoka-Higashiguchi, K., Morikawa, Y., \& Nakagawa, H. (2009). Relationship between Stress Coping and Burnout in Japanese Hospital Nurses. Journal of Nursing Management, 17, 359-365. http://dx.doi.org/10.1111/j.1365-2834.2008.00960.x

Selye, H. (1977). A Code for Coping with Stress. AORN Journal, 25, No 1.

Shimazu, A., Shimazu, M., \& Odara, T. (2009) Divergent Effects of Active Coping on Psychological Distress in the Context of the Job Demands-Control-Support Model: The Roles of Job Control and Social Support. International Journal of Behavioral Medicine, 12, 192-198. 